\title{
Editorial
}

\section{DNA damage and apoptosis}

\author{
JYJ Wang ${ }^{*, 1}$ \\ 1 Division of Biology and the Cancer Center, University of California, San Diego, \\ La Jolla, CA 92093-0322, USA \\ * Corresponding author: Tel: +1.858.534.6253; Fax: +1.858.534.2821; \\ E-mail: jywang@ucsd.edu
}

DNA damage is a common event in life, as evident by the continuous requirement of DNA repair to maintain genome integrity. The machinery of apoptosis is also common, present in a latent form in most if not all cells of multicellular organisms. A connection between DNA damage and apoptosis is supported by a large body of literature. However, the rules that govern this connection, which must be made judiciously, have remained unsolved.

What is very clear is that the tumor suppressor protein p53 provides an important link between DNA damage and apoptosis. In a succinct News and Commentary, Benchimol provides a comprehensive review on p53-regulated genes that are relevant to apoptosis. ${ }^{1}$ Thus far, none of the p53regulated and apoptosis-relevant genes, by themselves, can account for p53-mediated apoptosis in mammalian cells. ${ }^{1}$ By contrast, the Drosophila p53 (Dmp53) has been connected to Reaper, which plays a central role in DNA damage-induced apoptosis in the fly. ${ }^{2}$ The mammalian homolog of Reaper has not been reported, however, the mammalian Smac/Diablo appears to share the Reaper function. ${ }^{2}$ It is not known if the mammalian Smac/Diablo gene is a target of regulation by the mammalian p53. The already established upregulation of apoptosis inducers such as Bax, Noxa, Puma and p53AIP1 by $\mathrm{p} 53^{1}$ suggests that the Dmp53-Reaper paradigm is conserved in spirits, but may not directly apply to the mammalian p53.

Nordstrom and Abram $^{2}$ have noted that Dmp53 is equally homologous in its DNA binding domain to human p53 and p73. The mammalian p53-family contains three members, p53, p63 and p73. Cell Death and Differentiation has already dedicated its pages to a compendium of reviews on this family in December of $1999 .{ }^{3-6}$ The mammalian p73 is also involved in the regulation of apoptosis. The apoptotic function of p73 contributes to cell death induced by DNA damage, by E2F1, and by T-cell antigen receptor. ${ }^{3,7,8}$ The p73 gene also encodes an antiapoptosis protein, the $\Delta \mathrm{N}-\mathrm{p} 73 .^{9,10}$ Clearly, the mammalian p53 has to function within the context of its other family members, a situation that does not apply to the Dmp53. Exactly how the p53-family members coordinate their actions to regulate apoptosis is presently unknown.

The mechanisms that link DNA damage to the activation of p53 are also complex. The activation of p53 by ionizing radiation is better understood, and this is dependent on the ATM kinase. ${ }^{11}$ The ATM kinase functions as a transducer of DNA damage signal to $\mathrm{p} 53 .^{11}$ Ionizing radiation can activate the ATM kinase, possibly because ATM can bind to broken ends of DNA. ${ }^{11}$ The ATM and its related ATR kinase can also be activated by other types of DNA lesions. Cisplatin adducts in DNA, for example, are recognized by the mismatch repair (MMR) proteins, which appear to provide an essential link between platinated DNA and the ATM/ATR kinases. $^{12}$ The MMR proteins are important guardians of the genome, because they prevent the accumulation of spontaneous mutations generated during DNA replication. ${ }^{12}$ Obviously, MMR proteins do not signal apoptosis during each round of DNA replication. However, MMR proteins do activate apoptosis in response to cisplatin and other alkylating agents. ${ }^{12}$ Similarly, the ATM kinase can also stimulate DNA repair or apoptosis. In a majority of cells, ATM kinase offers important protection against ionizing radiation. However, with developing neurons, ATM kinase is required to activate apoptosis following ionizing radiation. ${ }^{11}$

Exactly how MMR proteins and ATM-kinase distinguish between their repair function versus their apoptosis signaling function is not understood. Because of their involvement in DNA repair, these proteins may 'sense' the efficiency or the status of DNA repair. Perhaps their function in signaling apoptosis is only switched on after they have 'sensed' failure in DNA repair. The notion that apoptosis is only activated after DNA repair fails is logically sound. However, there is no direct evidence supporting this hypothesis. Failure in DNA repair will cause cell death, even in yeast, which lacks the apoptosis program. Death from excessive DNA damage is passive, and should be experimentally distinguished from apoptosis, which is an active process.

The rules that govern the connection between DNA damage and apoptosis should operate on the already identified platform of lesion-sensors (MMR), signal transducers (ATM), and transcription regulation ( $p 53, p 73)$. It should be noted that this platform does not solely regulate apoptosis. The primary response to DNA damage is the stimulation of DNA repair and the activation of cell cycle checkpoints (Figure 1). The biological goal of this primary response is to protect the damaged cell. Apoptosis is a secondary response to DNA damage, with the biological goal of protecting a multicellular organism against a damaged cell. The p53-dependent transcription response can also trigger another secondary response to protect the organism, and this is to enforce the growth arrest of a damaged cell. The rules that govern DNA damage-induced apopotis, therefore, must be able to choose between p53dependent growth arrest versus apoptosis (Figure 1).

The choice between p53-dependent arrest and apoptosis can be made by two distinct, albeit not mutually exclusive, mechanisms. The first mechanism is to regulate p53 itself. The p53 protein that activates the growth arrest 


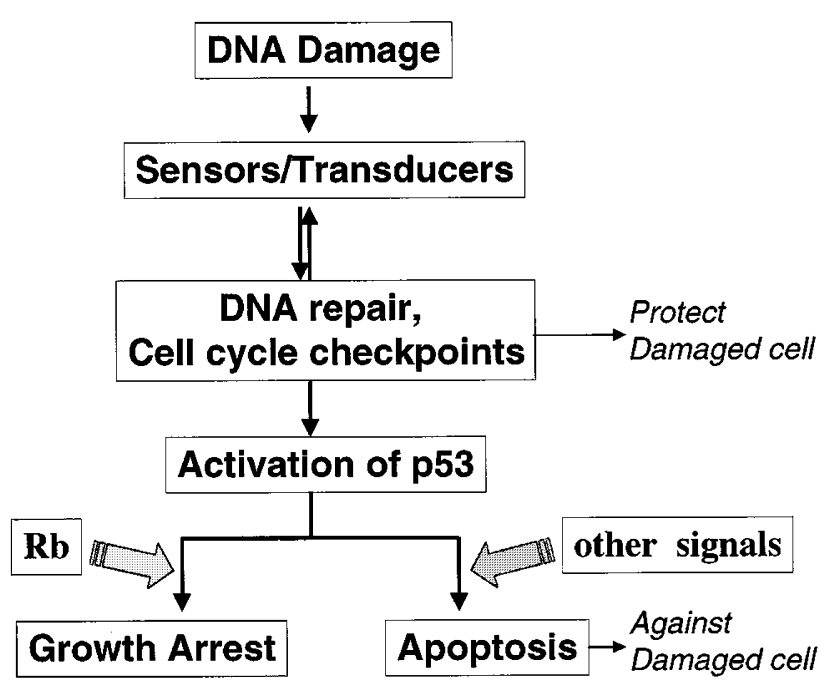

Figure 1 Apoptosis is one of several integrated responses to DNA damage. The activation of DNA repair and cell cycle checkpoints are conserved from unicellular to multicellular eukaryotes with the goal of protecting the damaged cell. The activation of $p 53$ and related family members can either enforce the cell cycle arrest or induce apoptosis. The p53-dependent responses are directed against the damaged cell to protect the organism. The rules that govern the choice between growth arrest and apoptosis are likely to be enforced by other proteins that can antagonize or synergize with p53 to regulate apoptosis

genes, e.g., p21Cip1, might be differentially modified from the p53 protein that activates the apoptosis genes, e.g., Bax, Noxa and Puma. The second mechanism is to regulate other proteins that can constrain the function of p53. For example, the retinoblastoma tumor suppressor protein $\mathrm{RB}$ is required for DNA damage-induced growth arrest. $^{13-15}$ Thus, the functional status of RB might determine whether a damaged cell chooses to arrest or to commit suicide. The pro-apoptotic proteins that are upregulated by p53 are also subjected to functional modulation by post-transcriptional mechanisms, ${ }^{16}$ which may also constrain the apoptosis function of p53.

The conundrum that p53 can activate a large number of genes, but none of them seems to play a deterministic role in apoptosis, supports the idea that p53 alone cannot specify the fate of apoptosis upon DNA damage. In the coming years, we expect to learn more about parallel pathways that either antagonize or synergize with p53 to regulate apoptosis in DNA damaged cells. In a forwardlooking review, El-Deiry discusses the interplay between p53- and TRAIL-regulated apoptosis pathways and their potential application to cancer therapy. ${ }^{17}$ The lesson biologists have learned repeatedly in complex systems is combinatorial specificity. The time has come to look beyond p53 for answers in DNA damage-induced apoptosis.

1. Benchimol S (2001) Cell Death Differ. 8: 1049-1051

2. Nordstrom W and Abram J (2000) Cell Death Differ. 7: 1035-1038

3. Levrero M et al (1999) Cell Death Differ. 6: 1146-1153

4. Ikawa $S$ et al (1999) Cell Death Differ. 6: 1154-1161

5. Lohrum MAE and Vousden KH (1999) Cell Death Differ. 6: 1162-1168

6. Arrowsmith $\mathrm{CH}$ (1999) Cell Death Differ. 6: 1169-1173

7. Irwin M et al (2000) Nature 407:645-648

8. Lissy NA et al (2000) Nature 407: 642-645

9. Pozniak CD et al (2000) Science 289: 304-306

10. Yang AN et al (2000) Nature 404: $99-103$

11. Khanna K et al (2001) Cell Death Differ 8: 1052-1065

12. Bellacosa A (2001) Cell Death Differ. 8: 1076-1092

13. Harrington EA et al (1998) Proc. Natl. Acad. USA 95: 11945-11950

14. Flatt PM et al (2000) Mol. Cell. Biol. 20: 4210-4223

15. Knudsen KE et al (2000) Mol. Cell. Biol. 20: 7751-7763

16. Korsmeyer SJ et al (1999) Cold Spring Harb. Symp. Quant. Biol. 64: 343-350

17. El-Deiry WS (2001) Cell Death Differ. 8: 1066-1075 